Check for updates

Cite this: RSC Adv., 2019, 9, 5901

\title{
Boron nitride nanochannels encapsulating a water/ heavy water layer for energy applications
}

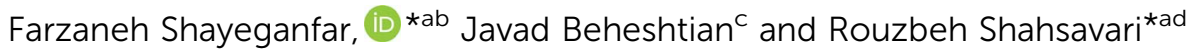

\begin{abstract}
Water interaction and transport through nanochannels of two-dimensional (2D) nanomaterials hold great promises in several applications including separation, energy harvesting and drug delivery. However, the fundamental underpinning of the electronic phenomena at the interface of such systems is poorly understood. Inspired by recent experiments, herein, we focus on water/heavy water in boron nitride (BN) nanochannels - as a model system - and report a series of ab initio based density functional theory (DFT) calculations on correlating the stability of adsorption and interfacial properties, decoding various synergies in the complex interfacial interactions of water encapsulated in BN nanocapillaries. We provide a comparison of phonon vibrational modes of water and heavy water $\left(D_{2} O\right)$ captured in bilayer $B N$ (BLBN) to compare their mobility and group speed as a key factor for separation mechanisms. This finding, combined with the fundamental insights into the nature of the interfacial properties, provides key hypotheses for the design of nanochannels.
\end{abstract}

Received 3rd December 2018 Accepted 6th February 2019

DOI: 10.1039/c8ra09925a

rsc.li/rsc-advances studied on electro/magneto modulated ion transport through GO membranes. While several experimental works demonstrate promising applications of nanochannels, the fundamental subatomic and electronic phenomena at the heart of such nanochannels are poorly understood.

Herein, we present a comprehensive ab initio study to decode the complex interfacial interactions of water and heavy water encapsulated in bilayer BN (BLBN) as a model system. Our findings demonstrated that $2 \mathrm{D} \mathrm{BN}$ can be used as nanochannels with tunable interlayer distance that is determined by in/outplane hydrogen bonds of water layers. To discover the mechanism for separating water and heavy water, we further investigate phonon spectrum for water layers between BLBN and for heavy water, deuterium oxide $\mathrm{D}_{2} \mathrm{O}$. This nanometer-scale $\mathrm{BN}$ capillaries is a flagship architecture in fabricating atomically channels for nanofluidic technology. ${ }^{\mathbf{1 8}}$

BN nanosheet could be a good candidate for water cleaning and purification as experimentally has been reported by Lei, et $a{ }^{19}{ }^{19}$ Moreover, Hao et al. in their paper ${ }^{20}$ reported the properties of atomic layer deposition (ALD) boron nitride nanotube, which is relevant for water purification. Li et al. ${ }^{21}$ reported that porous boron nitride nanosheets show an excellent performance for water purification.

More recently Falin et $a .^{22}$ synthesized single crystalline of mono/few layer BN nanosheets. They experimentally measured the Young's modulus of high quality 1-9 layer BN. ${ }^{21}$ Mechanical properties of $\mathrm{BN}$ nanosheets placed them among the strongest insulating materials, indicating the fracture strength of $70.5 \pm$ $5.5 \mathrm{GPa}^{21}$

To obtain fundamental insights on the interfacial interactions, a series of DFT calculations were performed on single
77005, USA. E-mail: rouzbeh@rice.edu; fs24@rice.edu

${ }^{b}$ Department of Energy Engineering and Physics, Amirkabir University of Technology, 14588 Tehran, Iran

${ }^{c}$ Department of Chemistry, Shahid Rajaee Teacher Training University, 16875-163 Tehran, Iran

${ }^{d}$ Department of Material Science and NanoEngineering, Rice University, Houston, TX 77005, USA 
layer water (SLW) molecules and double layer water (DLW) molecules settled on BN layers and encapsulated in BLBN (see Methods). We first examined the molecular models of SLW settled on BN layers with and without external field. Fig. 1a and b show SLW/BN, including $\left(\mathrm{H}_{2} \mathrm{O}\right)_{16}$, representing 16 water molecules and the substrate containing $120 \mathrm{~B}, \mathrm{~N}$ atoms. In order to take into account the effect of structural boundary conditions and compare the interfacial properties of SLW and DLW on BN surface, we employed the energy minimized structures for the SLW and DLW on BN layer (Fig. 1d and e), containing $32 \mathrm{H}_{2} \mathrm{O}$ molecules. The electrical dipole moment (Table 1) enhances with increased external electric field $E_{\text {ext }}$. For instance, the magnitude of dipole moment of SLW/BN system is 3.1 (debye) for $E_{\text {ext }}=0 \mathrm{~V} \AA^{-1}$, while it increases to 11.4 (debye) for $E_{\text {ext }}=0.3 \mathrm{~V}^{-1}$. Furthermore, the net charge transfer will increase as a direct result of increased dipole moment and $E_{\text {ext }}$.

Table 1 shows the DFT results in terms of the intermolecular energy $\left(E_{\text {int }}\right)$, the structural deformation $\left(d_{\mathrm{O}-\mathrm{H}}\right)$, the electrical dipole moment $\left(p_{z}\right)$, the intermediate pressure between adsorbate and surface $\left(P=\left(\Sigma_{\mathrm{i}, \mathrm{j}} F_{\mathrm{i}, \mathrm{j}}\right) / A\right.$, where $F_{\mathrm{i}, \mathrm{j}}$ is the force on ith atom of adsorbate due to jth atom of substrate and $A$ is the substrate area), energy gap $E_{\mathrm{g}}$ and lastly net charge on water layer.

In view of Table 1 , the following observations are in order: (i) SLW/BN is energetically more stable than DLW/BN, since the formation of several directional hydrogen bonds between the water molecules of DLW compensates the stability of the water layer on the $\mathrm{BN}$ substrate, (ii) the structural deformation for (a)

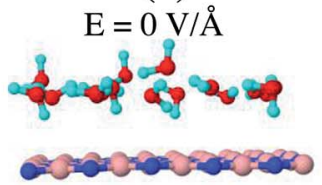

(b)

(c)

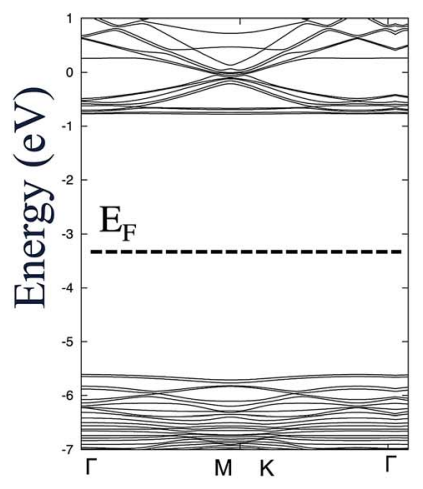

(d)

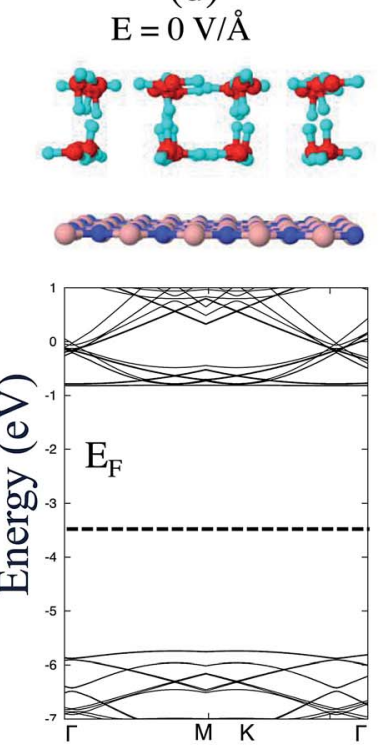

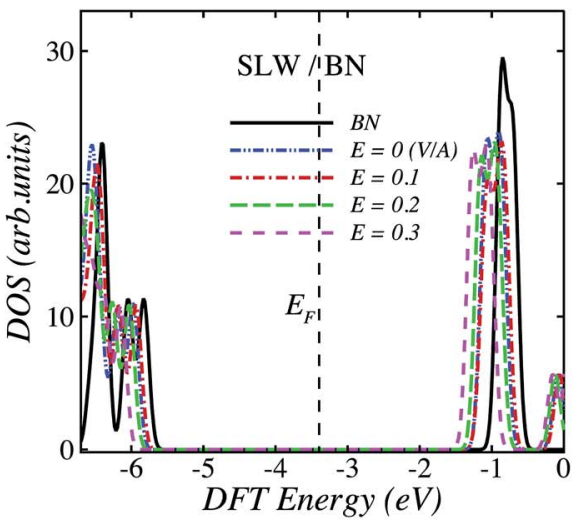

(e)
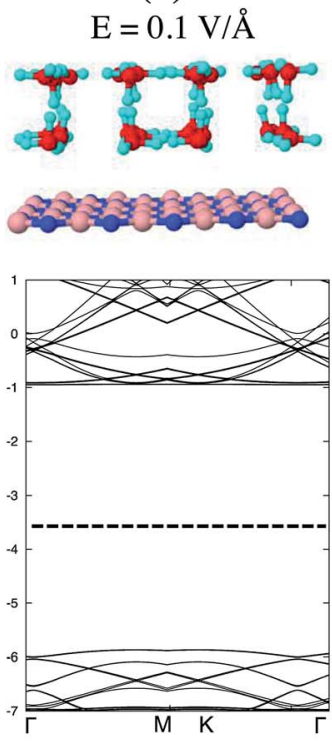

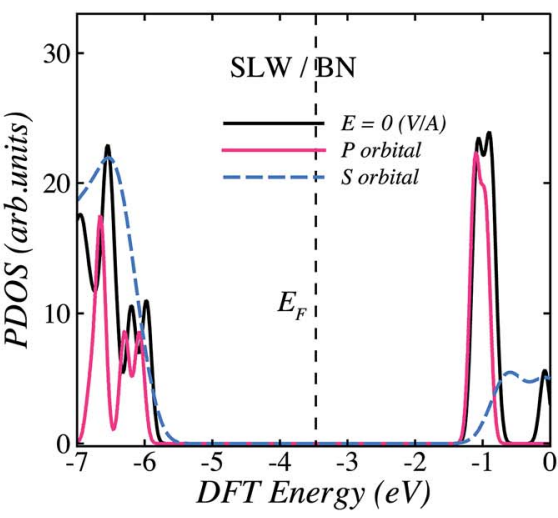

(f)

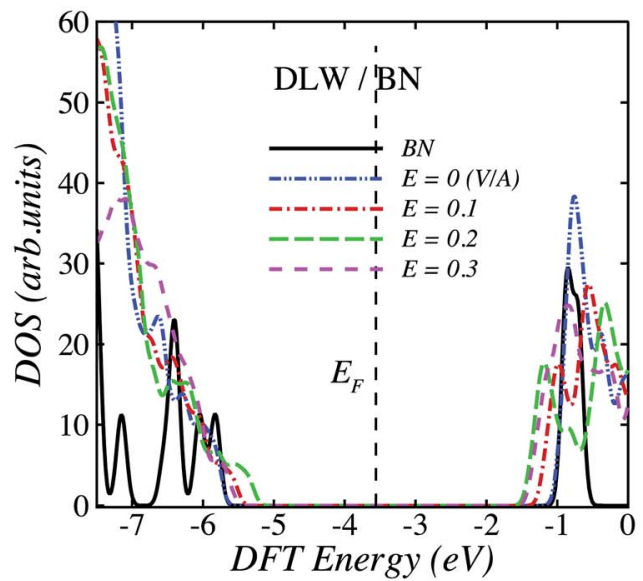

Fig. 1 Optimized single layer water (SLW)/BN and its electronic band structure for different external electric field, (a) $E=0 \vee \AA^{-1}$, (b) Total density of states (DOS) and (c) projected DOS for SLW/BN. Optimized double layer water (DLW)/BN and its electronic band structure for different external electric field, (d) $E=0$, (e) $E=0.1 \vee \AA^{-1}$, (f) total DOS for nanochannels of (a) and (b). 
Table 1 DFT results of interfacial properties of optimized SLW and DLW on BN

\begin{tabular}{|c|c|c|c|c|c|c|c|c|}
\hline & $\mathrm{SLW} / \mathrm{BN}$ & & & & $\mathrm{DLW} / \mathrm{BN}$ & & & \\
\hline$E_{\text {int }}(\mathrm{eV})$ & -2.41 & -2.57 & -2.73 & -2.95 & -2.11 & -2.23 & -2.37 & -2.53 \\
\hline Deformation (BN) $(\AA)$ & 0.1 & 0.13 & 0.15 & 0.17 & 0.033 & 0.098 & 0.11 & 0.14 \\
\hline Electrical dipole $\left(p_{\mathrm{z}}\right)$ (debye) & 3.1 & 7 & 9.3 & 11.4 & 2 & 5 & 8 & 11 \\
\hline Pressure (GPa) & 0.17 & 0.13 & 0.1 & 0.08 & 0.5 & 0.41 & 0.24 & 0.16 \\
\hline
\end{tabular}

SLW/BN is greater than DLW/BN due to the strength of intermolecular interaction, (iii) the vdW pressure for the stable system i.e., SLW/BN is $0.17 \mathrm{GPa}$, which is less than DLW/BN $0.5 \mathrm{GPa}$. To obtain deep insights on electronic properties of SLW/BN and DLW/BN, Fig. 1 shows the electronic band structure and total density of states (DOS) of these systems.

Fig. 1 b shows that DOS of SLW/BN has an energy shift while the presence of SLW does not significantly affect the electron density, confirming physisorption of SLW/BN. ${ }^{23}$ This finding suggests that the interaction of water with monolayer BN is weak, consistent with the hydrophobicity of BN. The interfacial SLW can infuse positive charges on the monolayer $\mathrm{BN}$, i.e., some electrons from the valence band (VB) of BN are transferred to SLW because of the existence of $\mathrm{OH}$ groups. This charge induction cause the DOS of SLW/BN system to be shifted by $0.15 \mathrm{eV}$ below the Fermi energy of the isolated BN, indicating pdoping of the contacting BN.

The electron density of states are experimentally measured by using scanning tunneling microscopy (STM). Therefore, to analyze orbital contribution of designed nanochannel, we plotted projected density of state (PDOS) in Fig. 1c for SLW/BN. This figure (PDOS) reveals that the interfacial interaction of water and $\mathrm{BN}$ layer is dominated by P-orbitals.

An analogous DOS information can be obtained for DLW/ BN. Applying $E_{\text {ext }}$ in the system enhances the distance between $\mathrm{H}$ and $\mathrm{O}$ atoms $d_{\mathrm{O}-\mathrm{H}}$. For instance, when $E_{\text {ext }}$ is $0.1 \mathrm{~V}$ $\AA^{-1}, d_{\mathrm{O}-\mathrm{H}}$ elongates to $1.0 \AA$, or $1.02 \AA$ for $E_{\text {ext }}=0: 2$ (Table 1).

Now we turn our attention to SLW and DLW encapsulated in BLBN. We are interested in comparing the variation of interfacial properties induced by SLW and DLW encapsulated between BLBN. Two structures were studied in Fig. 2 with and without applying $E_{\text {ext }}$. In order to clarify the influence of BLBN on the interfacial properties of water layers, we are presenting electronic band structure and DOS for SLW (DLW)@BLBN in Fig. 2. DFT results of the interfacial properties of these systems are presented in Table 2. The presence of $E_{\text {ext }}$ has not significantly affected the interactions of SLW with the bilayer surfaces. Nevertheless, the interfacial properties of water layer on monolayer and bilayer BN model indicates that these water layers constitute stable arrangement. This suggests that the use of BLBN would be advantageous in describing the mass transport interactions in nanochannel systems.

Comparing the intermolecular energy $\left(E_{\text {int }}\right)$ and vdW pressure of SLW or DLW @BLBN in Table 2, we find that the vdW pressure is more important for DLW@BLBN (0.42 GPa) given that DLW is less stable $\left(E_{\text {int }}=-0.6 \mathrm{eV}\right)$. This finding indicates that vdW pressure in nanoscale system plays a key role to tune their electronic properties.

Fig. $2 \mathrm{~b}$ describes the total DOS, while Fig. $2 \mathrm{c}$ shows the orbital contribution of SLW@BLBN. Again, one can observe that P-orbital is dominating over S-orbital for BLBN and confirms our obtained electronic properties. For instance, SLW@BLBN system shows $4.3 \mathrm{eV}$ band gap smaller than SLW/BN (4.8 eV), arising from hybridization of S-orbital and P-orbital and creating $\pi$ bond in BLBN weaker than SLW/BN. This weaker $\pi$ bond yields to correlation of electrons in conduction and valance band, creating smaller band gap.

Fig. 2f shows a few Fermi energy shift for 2D water networks encapsulated in BLBN with $E_{\text {ext }}$ and small differences in their electronic properties. The band structure and DOS associated with the SLW@BLBN (Fig. 2a-c) support the adsorption of SLW, suggesting that the weakly dispersed states in the BN can be linked to the hydrogen networks between the water molecules.

Table 2 shows several contrasting features in the optimized structural parameters of DLW@BLBN. As an example, increasing electric field in DLW@BLBN leads to the band gap closing from 4.5 to $3.0 \mathrm{eV}$. This bandgap closing may significantly contribute to the magnitude of enhanced electric dipole moment due to the external electric field.

To identify the source of band gap closing in DLW@BLBN, we examine the profile of electronic band structure in Fig. 2d and e. Dispersed bands in conduction bands (CB) (Fig. 2e) indicate increasing group velocity and electron mobility, imparting the polar character in these structures and band gap reduction, as reported previously. ${ }^{24}$ In particular, a slight displacement of the VB and CB toward Fermi level region is observed with increasing the electric field (Fig. 2f). ${ }^{25}$

Finally, we study the double layer heavy water (deuterium oxide) (DLD) between BLBN (Fig. 3). Phonon dispersion plots of isolated DLW (as a reference) and DLW encapsulated in BLBN are depicted in Fig. 3a and b, respectively. Fig. 3b shows that variation of the vibrational phonon modes of DLW@ BLBN includes additional details on the intermolecular interaction.

First and foremost, no negative phonon vibrations exist for both cases. Second, we observe that splitting occurs for long wavelengths of DLW@BLBN, as compared to isolated DLW. The origin of this splitting is the interior electric filed created by charge transfer in such system, in line with previous reports for ice systems. ${ }^{22}$ Simply put, the charge transfer between DLW and BLBN induces interior electric field, arising from ionic 
(a)
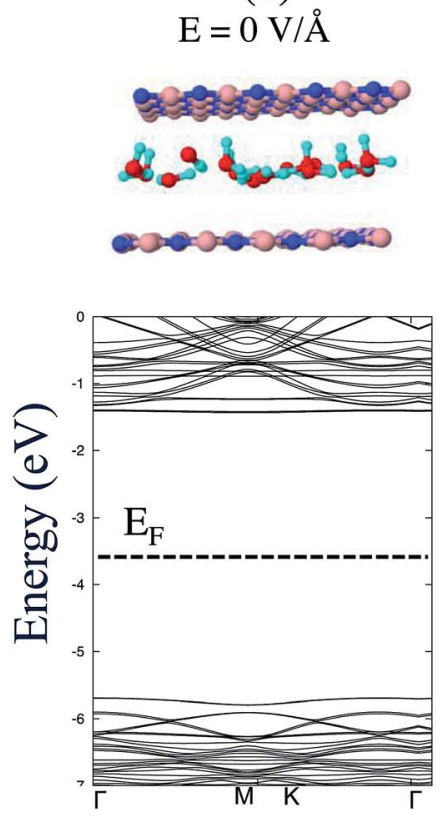

(d)

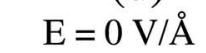

0,5202020

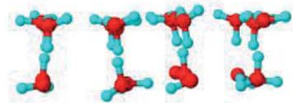

caicurasussoss
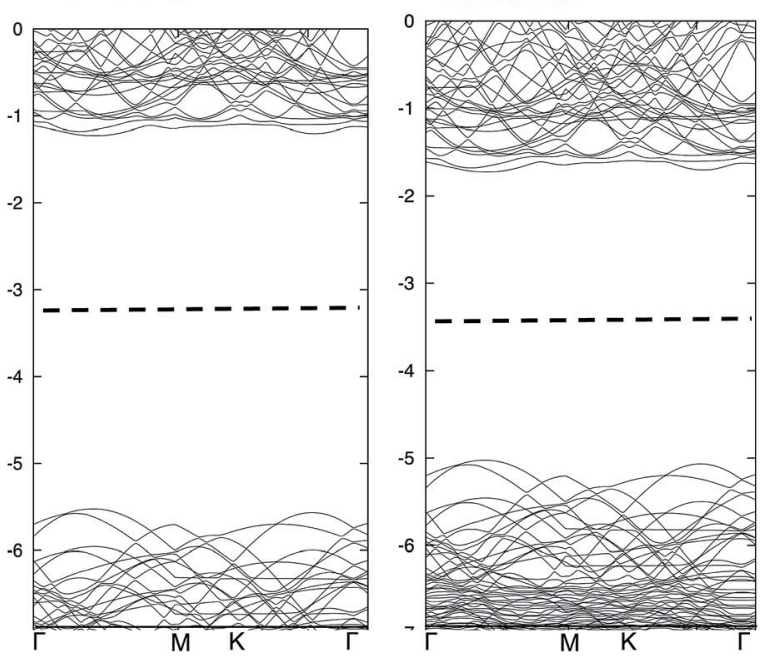

(b)

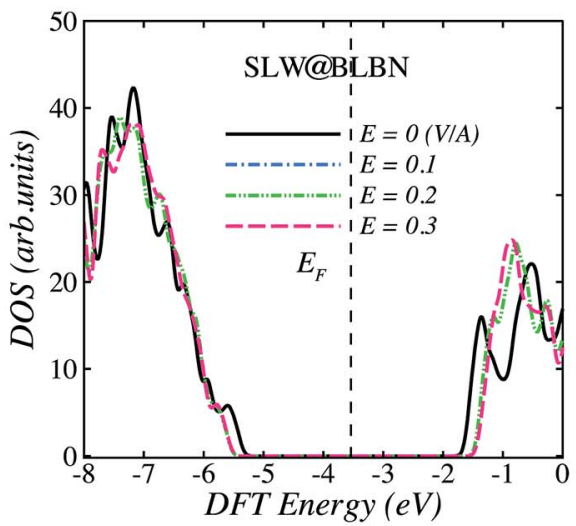

(e)

(c)

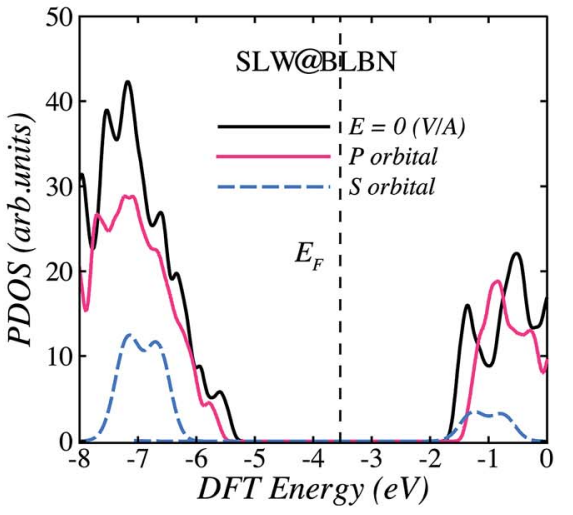

(f)

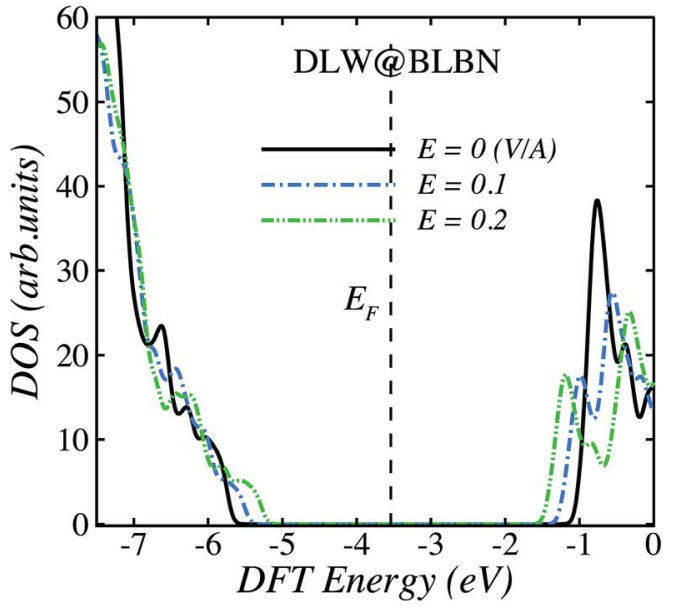

Fig. 2 Optimized single layer water (SLW)@BLBN and its electronic band structure for different external electric field, (a) $E=0 \vee \AA^{-1}$, (b) total density of states (DOS) and (c) projected DOS for nanochannels of (a). Optimized double layer water (DLW)@BLBN and its electronic band structure for different external electric field, (d) $E=0$, (e) $E=0.1 \vee \AA^{-1}$, (f) total DOS for nanochannels of (a) and (b).

displacements. Then, the long range Coulomb interactions between DLW water with BLBN cause splitting of the long wavelength. Third, there is some stop bands (bandgaps) in frequency spectra due to non-unity mass ratio of DLW. ${ }^{\mathbf{2 6}}$ The dispersion of bands is strongly influenced by intermolecular interaction of DLW trapped inside BLBN, while the stop bands are shifted up. Note that the acoustic phonon modes (small frequency extent) are dispersed for DLW@BLBN due to the interactions of DLW with localized electrons of BN.

These plots confirm that disperse bands lead to higher carrier mobility in contrast to localized carriers presented by flat bands. ${ }^{27} \mathrm{~A}$ common trend in these phonon spectra is that the 
Table 2 DFT results of the structural and interfacial properties of optimized SLW and DLW between BLBN

\begin{tabular}{|c|c|c|c|c|c|c|c|}
\hline \multicolumn{4}{|c|}{ SLW/BLBN } & \multicolumn{4}{|c|}{ DLW/BLBN } \\
\hline External electric field $E_{\text {ext }}\left(\mathrm{V} \AA^{-1}\right)$ & 0.0 & 0.1 & 0.2 & 0.3 & 0.0 & 0.1 & 0.2 \\
\hline$E_{\text {int }}(\mathrm{eV})$ & -1.96 & -2.04 & -2.18 & -2.78 & -0.6 & -1.43 & -1.62 \\
\hline Deformation (BN) (̊̊) & 0.13 & 0.15 & 0.15 & 0.14 & 0.8 & 0.97 & 0.96 \\
\hline Electrical dipole $\left(p_{\mathrm{z}}\right)$ (debye) & 4.5 & 8.3 & 12.3 & 28.3 & 0.07 & 4.6 & 9.6 \\
\hline Pressure (GPa) & 0.24 & 0.18 & 0.12 & 0.09 & 0.42 & 0.28 & 0.09 \\
\hline
\end{tabular}

contribution of optical phonons (large frequency extent) to the intermolecular interaction is negligible due to the flat bands, considering the low phonon group velocity.

Fig. 3d presents our results for phonon dispersion of double layer deuterium oxide (heavy water) (DLD)@BLBN, and Fig. 3c extends our findings for isolated DLD. For isolated DLD, we observe the expected phonon spectra data as experimentally reported. Interestingly, comparison of Fig. $3 \mathrm{c}$ and $\mathrm{d}$ indicate that the phonon modes differ for isolated DLD and DLW. Different trends can be secured from these phonon plots.

The first significant result lies in comparison of isolated DLD (Fig. 3c) and phonon spectra of DLD between BLBN (Fig. 3d), which is not drastically affected. The perceived reason can be considered as greater closed shell character of deuterium oxide than water, causing weaker interaction between DLD@BLBN than DLW@BLBN. The number of flat bands increases for large frequencies, indicating more localized carrier. ${ }^{28}$ Another consequence of phonon spectra is the distribution of stop bands in Fig. 3d, which differs from that of isolated DLD where the stop bands are shifted up.

Our results for deuterium oxide and water interaction are in line with experimental reports on scalable methods for hydrogen isotopes enrichment, due to the high level energy barrier of deuterons compared to proton. ${ }^{29}$ For instance, it is reported that the mobility of hydrogen is greater than deuterium ions in parent gases ${ }^{30}$ or deuterons permeate through BN nanostructures more slowly than protons, achieving a separation factor of $\sim 10{ }^{28}$ Exclusive properties of water encapsulated between 2D materials such as BN could lead to wide applications of nanochannels (e.g., separation, drug delivery, energy harvesting) by making nanometer-sized capillaries. Overall, these fundamental insights could be useful to understand, correlate and predict experimental results.

In summary, we investigated the interfacial and electronic properties of single/double layer water and heavy water settled on monolayer and double layer BN using DFT calculations. The

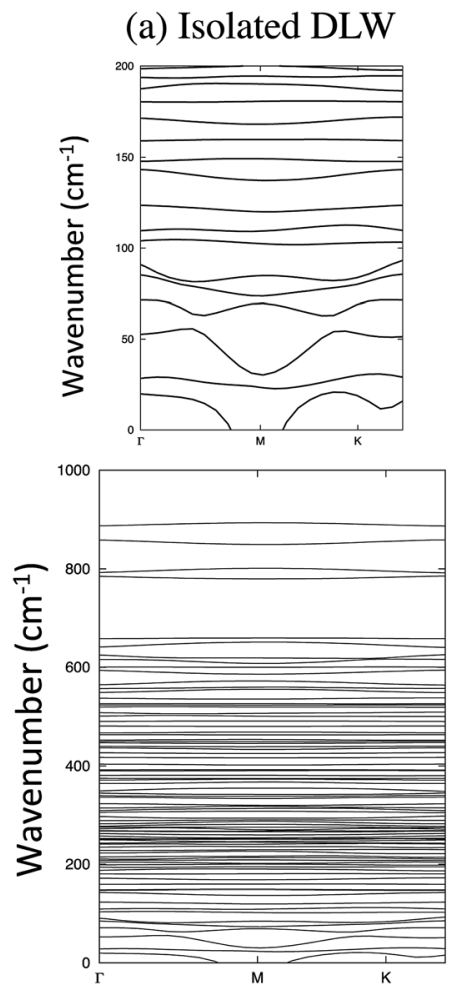

\section{(b) DLW @ BLBN}
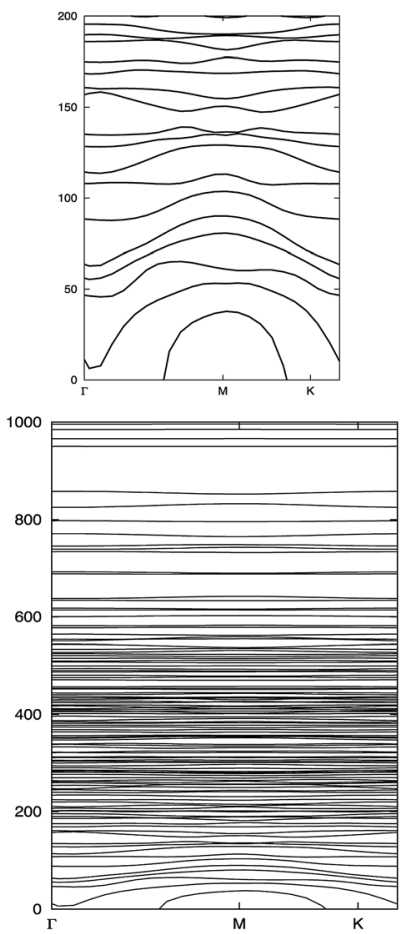

(c) Isolated DLD
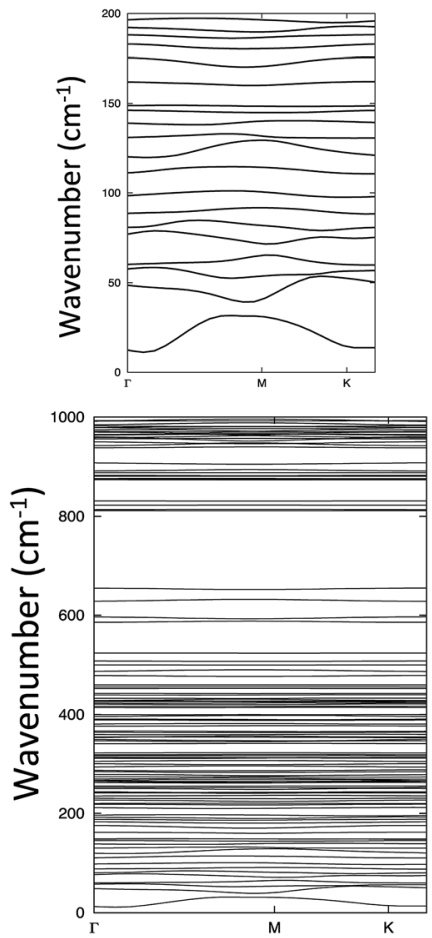

(d) DLD @ BLBN
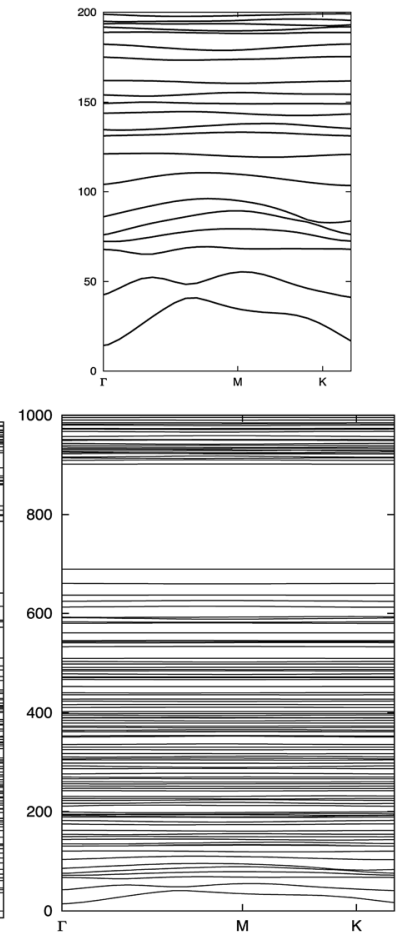

Fig. 3 Phonon spectrum for (a) isolated double layer water (DLW), (b) DLW@BLBN, (c) isolated deuterium oxide (heavy water) (DLD) and (d) DLD@BLBN. 
formation of DLW as a network reduces the extent of $\mathrm{OH}$ deformation, and their interfacial interactions on BN become less prevalent. Thus, SLW/BN is energetically more stable than $\mathrm{DLW} / \mathrm{BN}$. The formation of several directional hydrogen bonds between the water molecules in DLW compensates the stability of the water layer on the BN substrate. The phonon spectra of DLW@BLBN have strong dispersion related to their high mobility and group speed, supporting higher rate of water interaction through BLBN, in line with experiments. ${ }^{28}$ Considering deuterium oxide, the phonon bands are less dispersed than isolated water spectra, suggesting that slower DLD mobility than water, thus an effective separation tool for $\mathrm{D}_{2} \mathrm{O}$ and $\mathrm{H}_{2} \mathrm{O}$ through such $2 \mathrm{D}$ materials. Moreover, phonon spectra of DLW@BLBN reveals that high mobility of water inside BLBN is originated from the weak interaction of water with BLBN. Broadly, the findings and methods of this work can have implications on fundamental understanding and designing effective filtration tools using a host of other 2D mono- and multi-layer atomic sheets (e.g. molybdenum disulfide, niobium diselenide, layered double hydroxides, etc.) for separation, drug delivery, and energy harvesting.

\section{Methods}

To describe the structural, electronic and interfacial properties of single layer water (SLW) and double layer water (DLW) settled on monolayer BN and between BLBN, density functional theory (DFT) is used, via the projector augmented-wave (PAW) formalism ${ }^{31}$ by using VASP simulation package. ${ }^{32}$

To account the dispersion interaction, generalized gradient (GG) approximation associated with vdW-DF2 for exchangecorrelation (XC) energies was performed, which is based on the algorithm of Román-Pérez and Soler ${ }^{33}$ and refitted PerdewWang 86 (rPW86). ${ }^{34}$ Plane wave basis set and norm conserving Troullier-Martins pseudopotentials was employed to solve Kohn-Sham equations. We adopt a $12 \times 12 \times 1$ MonkhorstPack sampling grid, regarding the $k$-points for all structures. ${ }^{35}$ Two criteria were set to optimize total energy and force, $10^{-5} \mathrm{eV}$ and $0.01 \mathrm{eV}^{-1}$ respectively. ${ }^{22}$

Fig. 2 shows the supercell of water within BN layers, containing $240 \mathrm{~B}, \mathrm{~N}$ atoms of BLBN and $32 \mathrm{H}_{2} \mathrm{O}$ molecules (whose 32 $\mathrm{O}$ atoms and $64 \mathrm{H}$ atoms). The intermolecular energy of monolayer and double layer water on the surfaces, $E_{\text {int }}$, is defined by

$E_{\text {int }}=E($ water layer/substrate $)-E($ substrate $)+E($ water layer $)(1)$

where $E$ (water layer/substrate) is the total energy for energy minimized water layer on substrate, $E$ (substrate) is the total energy for the substrate supercell, $E$ (water layer) is the total energy of the isolated water layer in the supercell. The phonon spectra of SLW and DLW within BN layers are calculated by using VASP phonon calculation. To understand vibrational modes of our case study, the first step is to calculate linear response to replacing phonon frequency vibrations in the real space. ${ }^{33}$ Dynamical matrices are computed at ten wave $(\sim \boldsymbol{q})$ vectors in the irreducible wedge of brillouin zone (BZ), i.e., on 10 $\times 10 \times 10$ grid. $^{34}$

\section{Conflicts of interest}

The authors declare no competing financial interest.

\section{Acknowledgements}

The supercomputer machines utilized in this work were supported in part by NIH award NCRR S10RR02950 and an IBM Shared Rice University Research (SUR).

\section{References}

1 L. Ci, L. Song, C. H. Jin, D. Jariwala, D. X. Wu, Y. J. Li, A. Srivastava, Z. F. Wang, K. Storr, L. Balicas, F. Liu and P. M. Ajayan, Atomic layers of hybridized boron nitride and graphene domains, Nat. Mater., 2010, 9, 430.

2 L. Song, L. Ci, H. Lu, P. B. Sorokin, C. Jin, J. Ni, A. G. Kvashnin, D. G. Kvashnin, J. Lou, B. I. Yakobson and P. M. Ajayan, Large Scale Growth and Characterization of Atomic Hexagonal Boron Nitride Layers, Nano Lett., 2010, 10(8), 3209-3215.

3 M. J. Rand and J. F. Roberts, Preparation and Properties of Thin Film Boron Nitride, J. Electrochem. Soc., 1968, 115, 423.

4 K. Miyoshi, D. H. Buckley, J. J. Pouch, S. A. Alterovitz and H. E. Sliney, Mechanical Strength and Tribological Behavior of Ion-Beam Deposited Boron Nitride Films on Non-Metallic Substrates, Surf. Coat. Technol., 1987, 33, 221.

5 L. H. Li, J. Cervenka, K. Watanabe, T. Taniguchi and Y. Chen, Strong Oxidation Resistance of Atomically Thin Boron Nitride Nanosheets, ACS Nano, 2014, 8, 1457.

6 C. Jin, F. Lin, K. Suenaga and S. Iijima, Fabrication of a Freestanding Boron Nitride Single Layer and Its Defect Assignments, Phys. Rev. Lett., 2009, 102, 195505.

7 C. Zhi, Y. Bando, C. Tang, H. Kuwahara and D. Golberg, Large Scale Fabrication of Boron Nitride Nanosheets and Their Utilization in Polymeric Composites with Improved Thermal and Mechanical Properties, Adv. Mater., 2009, 21, 2889-2893.

8 L. Cao, S. Emami and K. Lafdi, Large-Scale Exfoliation of Hexagonal Boron Nitride Nanosheets in Liquid Phase, Mater. Express, 2014, 4, 165-171.

9 J. Zhu, H. Wang, J. Liu, L. Ouyang and M. Zhu, Exfoliation of $\mathrm{MoS}_{2}$ and h-BN Nanosheets by Hydrolysis of $\mathrm{LiBH}_{4}$, Nanotechnology, 2017, 28, 115604.

10 H. Feng, Z. Hua and X. Liu, Facile and Efficient Exfoliation of Inorganic Layered Materials Using Liquid Alkali Metal Alloys, Chem. Commun., 2015, 51, 10961-10964.

11 R. K. Joshi, P. Carbone, F. C. Wang, V. G. Kravets, Y. Su, I. V. Grigorieva, H. A. Wu, A. K. Geim and R. R. Nair, Precise and Ultrafast Molecular Sieving Through Graphene Oxide Membranes, Science, 2014, 343, 752.

12 J. Abraham, K. S. Vasu, C. D. Williams, K. Gopinadhan, Y. Su, C. Cherian, J. Dix, E. Prestat, S. J. Haigh, I. V. Grigorieva, P. Carbone, A. K. Geim and R. R. Nair, Tunable Sieving of Ions Using Graphene Oxide Membranes, Nat. Nanotechnol., 2017, 12, 546-550. 
13 K. V. Agrawal, S. Shimizu, L. W. Drahushuk, D. Kilcoyne and M. S. Strano, Observation of Extreme Phase Transition Temperatures of Water Confined Inside Isolated Carbon Nanotube, Nat. Nanotechnol., 2017, 12, 267-273.

14 B. Radha, A. Esfandiar, F. C. Wang, A. P. Rooney, K. Gopinadhan, A. Keerthi, A. Mishchenko, A. Janardanan, P. Blake, L. Fumagalli, M. Lozada-Hidalgo, S. Garaj, S. J. Haigh, I. V. Grigorieva, H. A. Wu and A. K. Geim, Molecular Transport Through Capillaries Made with Atomic-Scale Precision, Nature, 2016, 538, 222-225.

15 M. J. Shultz, T. H. Vu, B. Meyer and P. Bisson, Water: A Responsive Small Molecule, Acc. Chem. Res., 2012, 45, 15-22.

16 V. Vaida, H. G. Kjaergaard, P. E. Hintze and D. J. Donaldson, Photolysis of Sulfuric Acid Vapor by Visible Solar Radiation, Science, 2003, 299, 1566-1568.

17 P. Sun, F. Zheng, K. Wang, M. Zhong, D. Wu and H. Zhu, Electro- and Magneto-Modulated Ion Transport Through Graphene Oxide Membranes, Sci. Rep., 2014, 4, 6798.

18 A. Morelos-Gomez, R. Cruz-Silva, H. Muramatsu, J. OrtizMedina, T. Araki, T. Fukuyo, S. Tejima, K. Takeuchi, T. Hayashi, M. Terrones and M. Endo, Effective $\mathrm{NaCl}$ and Dye Rejection of Hybrid Graphene Oxide/Graphene Layered Membranes, Nat. Nanotechnol., 2017, 12, 1083-1088.

19 W. Lei, D. Portehault, D. Liu, S. Qin and Y. Chen, Porous boron nitride nanosheets for effective water cleaning, Nat. Commun., 2013, 4, 1777.

$20 \mathrm{~W}$. Hao, C. Marichy and A. Brioude, Promising properties of ALD boron nitride nanotube mats for water purification, Environ. Sci.: Nano, 2017, 4, 2311-2320.

21 J. Li, P. Jin, W. Dai, C. Wang, R. Li, T. Wu and C. Tang, Excellent performance for water purification achieved by activated porous boron nitride nanosheets, Mater. Chem. Phys., 2017, 196, 186-193.

22 A. Falin, Q. Cai, J. G. Elton, D. Scullion, D. Qian, R. Zhang, Z. Yang, S. Huang, K. Watanabe, T. Taniguchi, M. R. Barnett, Y. Chen, R. S. Ruoff and L. H. Li, Mechanical properties of atomically thin boron nitride and the role of interlayer interactions, Nat. Commun., 2017, 8, 15815.

23 F. Shayeganfar and R. Shahsavar, Electronic and pseudomagnetic properties of hybrid carbon/boron-nitride nanomaterials via $a b$ initio calculations and elasticity theory, Carbon, 2016, 99, 523-532.

24 Z. Zhang, W. Guoa and B. I. Yakobson, Self-Modulated Band Gap in Boron Nitride Nanoribbons and Hydrogenated Sheets, Nanoscale, 2013, 5, 6381-6387.

25 F. Shayeganfar and A. Rochefort, Tuning the Electronic Properties of a Boron-Doped $\mathrm{Si}(111)$ Surface by SelfAssembling of Trimesic Acid, J. Phys. Chem. C, 2015, 119(27), 1574215748.

26 A. J. H. McGaughey, M. I. Husseinz, E. S. Landry, M. Kaviany and G. M. Hulbert, Phys. Rev. B: Condens. Matter Mater. Phys., 2006, 74, 104304.

27 F. Shayeganfar and R. Shahsavari, Electronic and Pseudomagnetic Properties of Hybrid Carbon/BoronNitride Nanomaterials via $A b$ Initio Calculations and Elasticity Theory, Carbon, 2016, 99, 523-532.

28 F. Shayeganfar, J. Beheshtian and R. Shahsavari, FirstPrinciples Study of Water Nanotubes Captured Inside Carbon/Boron Nitride Nanotubes, Langmuir, 2018, 34(37), 11176-11187.

29 M. Lozada-Hidalgo, S. Hu, O. Marshall, A. Mishchenko, A. N. Grigorenko, R. A. W. Dryfe, B. Radha, I. V. Grigorieva and A. K. Geim, Sieving Hydrogen Isotopes Through TwoDimensional Crystals, Science, 2016, 351, 6268.

30 D. J. Rose, Mobility of Hydrogen and Deuterium Positive Ions in their Parent Gases, J. Appl. Phys., 1960, 31, 643.

31 P. E. Blchl, Projector Augmented-Wave Method, Phys. Rev. B: Condens. Matter Mater. Phys., 1994, 50, 17953.

32 G. Kresse and J. Hafner, Ab Initio Molecular Dynamics for Liquid Metals, Phys. Rev. B: Condens. Matter Mater. Phys., 1993, 47, 558.

33 G. Román-Pérez and J. M. Soler, Efficient Implementation of a van der Waals Density Functional: Application to DoubleWall Carbon Nanotubes, Phys. Rev. Lett., 2009, 103, 096102.

34 E. D. Murray, K. Lee and D. C. Langreth, Investigation of Exchange Energy Density Functional Accuracy for Interacting Molecules, J. Chem. Theory Comput., 2009, 5, 2754 .

35 F. Shayeganfar, K. S. Vasu, R. R. Nair, F. M. Peeters and M. Neek-Amal, Monolayer Alkali and Transition Metal Monoxides: MgO, CaO, MnO and NiO, Phys. Rev. B, 2017, 99, 523-532. 